quential topology $\tau$ defined on $L$ will be called "continuous" if and only if it is continuous in the operations of the system - that is,

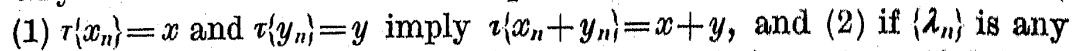
sequence of real numbers converging to $\lambda$, and $\tau\left\{x_{n}\right\}=x$, then $\tau\left\{\lambda_{n} x_{n}\right\}=x$. It is clear that if $x_{n} \equiv x$ implies $\tau\left\{x_{n}\right\}=x$, then $\tau\left\{x_{n}\right\}=x$ if and only if $z\left\{x_{n}-x\right\}=\Theta$. This will be assumed throughout $\S 7$.

Theorem 16: The join of any number of continuous sequential topologies is itself continuous.

The proof is that sketched for Theorem 6 .

Theorem 1\%: The most inclusive continuous sequential topology $\tau^{\prime \prime}$ included in each of two given continuous sequential topologies $\tau$ and $\tau^{\prime}$ exists and is given by the rule $\boldsymbol{\tau}^{\prime \prime}\left\{x_{n}\right\}=\Theta$ if and only if $\left\{y_{n}\right\}$ and $\left\{z_{n}\right\}$ exist, satisfying $x_{n}=y_{n}+z_{n}$ and $\tau\left\{y_{n}\right\}=\tau^{\prime}\left\{z_{n}\right\}=\Theta$.

The only real question is as to whether this rule defines a continuous sequential topology; if it does, $\tau^{\prime \prime} \subset \tau$ and $\tau^{\prime \prime} \subset \tau^{\prime}$ are obvious, and is also obvious that any continuous sequential topology including $\tau$ and $\boldsymbol{r}^{\prime}$ must include $\boldsymbol{\tau}^{\prime \prime}$. Further, $\boldsymbol{\tau}^{\prime \prime}$ and homogeneity evidently define a unique sequential topology.

Now suppose $\tau^{\prime \prime}\left\{x_{n}\right\}=$ and $\tau^{\prime \prime}\left\{x_{n}^{\prime}\right\}=x^{\prime}$. Then we can find $\left\{y_{n}\right\}$ $\left\{z_{n},\left\{y_{n}^{\prime}\right\}\right.$ and $\left\{z_{n}^{\prime}\right\}$ such that $y_{n}+z_{n}=x_{n}-x, y_{n}^{\prime}+z_{n}^{\prime}=x_{n}^{\prime}-x^{\prime}$, $\tau\left\{y_{n}\right\}=\tau^{\prime}\left\{z_{n\}}\right\}=\tau\left\{y_{n}^{\prime}\right\}=\tau^{\prime}\left\{z_{n}^{\prime}\right\}=\Theta$. Hence $\tau\left\{y_{n}+y_{n\}}^{\prime}\right\}=\tau^{\prime}\left\{z_{n}+z_{n}^{\prime}\right\}=\Theta$, by definition

$$
\tau^{\prime \prime}\left\{\left(x_{n}+x_{n}^{\prime}\right)-\left(x+x^{\prime}\right)\right\}=\tau^{\prime \prime}\left\{\left(y_{n}+y_{n}^{\prime}\right)+\left(z_{n}+z_{n}^{\prime}\right)\right\}=\Theta
$$

and $\tau^{\prime \prime}\left\{x_{n}+x_{n}^{\prime}\right\}=x+x^{\prime}$. The proof that $\lambda_{n} \rightarrow \lambda$ and $\boldsymbol{u}^{\prime \prime}\left\{x_{n}\right\}=x$ implies $\tau^{\prime \prime}\left(\lambda_{n} x_{n}\right\}=\lambda x$ follows similar lines.

Society of Fellows Harvard University.

\section{On the differentiation of additive functions of rectangles.}

By

\section{A. J. Ward (Cambridge).}

It has recently been shown that if $f(x, y)$ is a summable function of two variables, it is not in general true that

$$
\operatorname{Lim}_{d(R) \rightarrow 0} \frac{1}{\mu R} \iint_{R} f d x d y=f\left(x_{0}, y_{0}\right)
$$

almost everywhere, where $R$ is any rectangle containing the point $\left.\left.\left(x_{0}, y_{0}\right)^{x}\right)^{2}\right)$. Saks raised the question whether it is possible for the upper and lower limits, as $h, k \rightarrow 0$, of

$$
\frac{1}{4 h k} \int_{x_{0}-h}^{x_{0}+h} \int_{y_{0}-k}^{y_{y}+k} f(x, y) d x d y
$$

to be finite and not identical at the points of a set of positive measure $\left.{ }^{3}\right)$. Besicovitch has solved a slightly different but closely related problem by showing that

$$
\varlimsup_{h, k \rightarrow 0} \frac{1}{h k} \int_{x_{0}}^{x_{0}+h} \int_{y_{0}}^{y_{0}+k} f(x, y) d x d y
$$

is equal, at almost all points, either to $f\left(x_{0}, y_{0}\right)$ or to $\left.+\infty^{4}\right)$.

1) H. Busemann and W. Feller, Fund. Math. 22 (1934), 226-256; S. Saks, ibid., 257-261.

2) Here, as throughout this paper, $R$ denotes a closed rectangle with sides parallel to the co-ordinate axes, $d(R)$ its diameter, and $\mu$ Lebesgue plane measure.

3) Saks, loc. cit., 260

4) A. S. Besicovitch, Fund. Math. 25 (1935), 209-216. 
In this paper I consider the analogous limits obtained when the integral $\iint_{R} f(x, y) d x d y$ is replaced by an arbitrary additive function of rectangles $F(R)$. The theorems proved include the result of Besicovitch as a special case.

1. Let $F(x, y)$ be any real function of two real variables, defined in the interior of some square. Given any point $(x, y)$ and two numbers $h, k$ (which may be positive or negative but do not vanish), we write $R(x, y ; h ; k)$ for the rectangle whose opposite corners are $(x, y)$ and $(x+h, y+k)$. (All rectangles considered have their sides parallel to the coordinate axes.) We write

(1) $\Delta F(x, y ; h, k)=F(x+h, y+k)-F^{\prime}(x+h, y)-F(x, y+k)+F(x, y)$;

$$
F[R(x, y ; h, k)]=\Delta F(x, y ; h, k) \operatorname{sgn}(h k) .
$$

Thus for any rectangle $R, F(R)$ is defined, and has the same value whichever corner we take as $(x, y)$; also $F(R)$ is an additive function of rectangles. We define

$$
\bar{D}(x, y)=\varlimsup_{d(R) \rightarrow 0} \frac{F(R)}{\mu R}
$$

where $R$ is any rectangle containing $(x, y)$ either as an interior point or on the boundary. Since any rectangle containing $(x, y)$ can be divided into at most four rectangles, each of which has $(x, y)$ at one corner, we see from (2) and (3) that we have also

We define further

$$
\bar{D}(x, y)=\varlimsup_{h, k \rightarrow 0} \frac{\Delta F(x, y ; h, k)}{h k} .
$$

$$
\bar{D}_{m}(x, y)=\varlimsup_{h \rightarrow 0} \frac{\Delta F(x, y ; h, h)}{h^{2}} .
$$
There are similar definitions for the lower derivates $\underline{D}(x, y), \underline{D}_{m}(x, y)$.
We have at once

$$
\underline{D}(x, y) \leqslant \underline{D}_{m}(x, y) \leqslant \bar{D}_{m}(x, y) \leqslant \bar{D}(x, y) .
$$

2. We first state an elementary geometrical lemma. By a principal corner of any rectangle we mean either the lower leftmhand or the upper right-hand corner.
Lemma. If in any rectangle $R$ there is given a finite number of rectangles $R_{v}$, not touching or over-lapping each other or the sides of $R$; then the remaining area $R-\sum R_{y}$ can be divided into a finite number of rectangles $R_{\nu}^{\prime}$, each of which has as one of its corners a principal corner of one of the rectangles $R_{w}$.

The rectangles $R_{\nu}^{\prime}$ may be constructed thus: Produce each horizontal side of every rectangle $R_{v}$ as far as possible to the left, until it meets either the left-hand side of $R$ or the right-hand side of some other $R_{v}$. Then produce each right-hand vertical side of $R_{v}$ as far as possible, both ways, without crossing any of the horizontal lines already in existence. It may be shown ${ }^{5}$ ) that the lines thus drawn divide $R-\Sigma R_{\text {, }}$ into a set of rectangles $R^{\prime \prime}$, each of which contains a principal corner of some $R_{\nu}$, either at a corner on on a side. If on a side, we have merely to divide $R^{\prime \prime}$ into two parts by a line drawn from that point. Thus we obtain the required subdivision into rectangles $R_{\nu}^{\prime}$

3. Theorem 1. If at each point $(x, y)$ of a set $E$, we have $-\infty<D(x, y) \leqslant \bar{D}(x, y)<\infty$; then, almost everywhere in $E$, $\underline{D}(x, y)=\bar{D}(x, y)$.

We shall show that, almost everywhere in $E$,

$$
\underline{D}(x, y) \geqslant \bar{D}_{m}(x, y)
$$

Then by a similar argument we have almost everywhere in $E$

$$
\bar{D}(x, y) \leqslant \underline{D}_{m}(x, y)
$$

and combining these inequalities with (6) we obtain the desired result. Suppose on the contrary that in a subset of $\boldsymbol{E}$ of positive outer measure, we have

$$
-\infty<\underline{D}(x, y)<\bar{D}_{m}(x, y) \leqslant \bar{D}(x, y)<\infty .
$$

Then we can find $M$ so large and $\eta>0$ so small that in a set $E_{1}$, of positive outer measure we have

$$
-M<\underline{D}(x, y)<\bar{D}_{m}(x, y)-3 \eta<\bar{D}(x, y)<M .
$$

We can then find an integer $A$ such that in a set $E_{2}$ of positive outer measure, (8) holds and also

$$
\begin{gathered}
\underline{D}(x, y)<(A-2) \eta \\
\bar{D}_{m}(x, y)>A \eta
\end{gathered}
$$

s) The proof is easy and will not be given. 
From (8) (9) and (10) we see that

$$
|A \eta|<M \text {. }
$$

For any point $(x, y)$ of $E_{2}$, there exists $\delta(x, y)>0$ such that

$$
-M \leqslant \frac{F[R(x, y ; h, k)]}{|h| k \mid} \leqslant M
$$

whenever $0<|h|<\delta(x, y) ; 0<|k|<\delta(x, y)$; from (8). Hence, we can find a fixed $\delta>0$ and a set $E_{3}$, contained in $E_{2}$, of positive outer measure, such that (12) is true whenever $0<h|<\delta, \quad 0<| k \mid<\delta$, and $(x, y)$ lies in $E_{3}$. Let $E_{4}$ be the set of points of $E_{3}$ which are points of outer density of $E_{3}$ in the strong sense $\left.{ }^{\circ}\right)$. Then $\mu_{\theta} E_{4}=\mu_{e} E_{8}>0$.

4. Let $(x, y)$ be any point of $E_{4}$. We shall show that there exists a sequence of rectangles $R\left(x, y ; h_{n}, k_{n}\right), n=1,2, \ldots$ such that

$$
\begin{gathered}
h_{n} k_{n}>0, \quad \frac{1}{2}\left|k_{n}\right|<\left|h_{n}\right|<2\left|k_{n}\right|, \\
h_{n}, k_{n} \rightarrow 0 \quad \text { as } \quad n \rightarrow \infty, \\
\left(x+h_{n}, y+k_{n}\right) \quad \text { belongs to } E_{3}, \\
F\left[R\left(x, y ; h_{n}, k_{n}\right)\right]>(A-1) \eta h_{n} k_{n} .
\end{gathered}
$$

For from (10) and the fact that $(x, y)$ is a point of outer density of $E_{3}$, we can find a sequence of squares $S_{n}=R\left(x, y ; l_{n}, l_{n}\right)$ such that

\section{where}

$$
\begin{gathered}
l_{n} \mid<\delta \text { and } l_{n} \rightarrow 0 \text { as } n \rightarrow \infty, \\
F\left(S_{n}\right)>A \eta l_{n}^{2}
\end{gathered}
$$

$$
\varepsilon=\eta / 16 M .
$$

From (8) clearly $0<\varepsilon<\frac{1}{2}$. Consider one such square $S_{n}$, and suppose for example that $l_{n}>0$. By (19) there exist $h_{n}, k_{n}$ such that

$$
\begin{aligned}
& (1-\varepsilon) l_{n}<h_{n}<l_{n}, \\
& (1-\varepsilon) l_{n}<k_{n}<l_{n},
\end{aligned}
$$

3) The point $(x, y)$ is a point of outer density of set $x$, in the strong sense, if $\lim _{d(R) \rightarrow 0} \frac{\| !(E R)}{\iota^{\prime \prime}(R)}=1$, where $R$ is any rectangle, containing $(x, y)$, with sides parallel to the axes. S. Saks, Theorie de l'Intégrale, Warsaw 1933, pp. 223, 231. and $\left(x+h_{n}, y+k_{n}\right)$ belongs to $E_{3}$. We can express the rectangle $R\left(x, y ; h_{n}, k_{n}\right)$ as

$$
R\left(x, y ; h_{n}, k_{n}\right)=S_{n}-R_{n 1}-R_{n 2}-R_{n 3}
$$

where $R_{n 1}, R_{n 2}, R_{n 3}$ have each one corner at $\left(x+h_{n}, y+k_{n}\right)$ and their opposite corners at $\left(x, y+l_{n}\right) ;\left(x+l_{n}, y+l_{n}\right) ;\left(x+l_{n}, y\right)$ respectively. The sides of these rectangles are less than $l_{n}$ and so less than $\delta$; hence as $\left(x+h_{n}, y+k_{n}\right)$ belongs to $E_{3}$ we have from (12)

$$
F\left(R_{n 1}\right)+F\left(R_{n 2}\right)+F\left(R_{n 3}\right) \leqslant M \sum_{i=1}^{3} \mu\left(R_{n i}\right)
$$

and so from (18) (21) (22) (20) and (11), since $F(R)$ is additive,

$$
\begin{aligned}
F\left[R\left(x, y ; h_{n}, k_{n}\right)\right] & >A \eta l_{n}^{2}-M\left(l_{n}^{2}-h_{n} k_{n}\right) \\
& >A \eta h_{n} k_{n}-(M-A \eta)\left(l_{n}^{2}-h_{n} k_{n}\right) \\
& >A \eta h_{n} k_{n}-2 M l_{n}^{2}\left[1-(1-\varepsilon)^{2}\right] \\
& >A \eta h_{n} k_{n}-4 M \epsilon l_{n}^{2} \\
& >A \eta h_{n} k_{n}-\eta l_{n}^{2} / 4 \\
& >(A-1) \eta h_{n} k_{n}
\end{aligned}
$$

since from (21) and (22) we have certainly $h_{n} k_{n}>l_{n}^{2} / 4$, for $\varepsilon<\frac{1}{2}$. We have now shown that $h_{n}, k_{n}$ satisfy conditions (15) and (16). From (21) and (22), since $\varepsilon<\frac{1}{2},(13)$ is also satisfied. If $l_{n}<0$ we can proceed in a similar way; in this case $h_{n}$ and $k_{n}$ will be negative. Thus $h_{n}, k_{n}$ are defined for all $n$, and since $l_{n} \rightarrow 0$ we have clearly $h_{n}, k_{n} \rightarrow 0$ as $n \rightarrow \infty$.

5. Consider now a fixed point $\left(x_{0}, y_{0}\right)$ of $E_{4}$; since $E_{3}-E_{4}$ is of measure zero, $\left(x_{0}, y_{0}\right)$ is also a point of outer density of $E_{4}$ in the strong sense. From (9) we see that there exist $h_{0}, k_{0}$ such that, writing $R_{0}=R\left(x_{0}, y_{0} ; h_{0}, k_{0}\right)$,

$$
\begin{gathered}
F\left(R_{0}\right)<(A-2) \eta \mu\left(R_{0}\right), \\
0<\left|h_{0}\right|<\delta, \quad 0<\left|k_{0}\right|<\delta, \\
\mu_{e}\left(E_{4} R_{0}\right)>(1-\varepsilon) \mu\left(R_{0}\right) .
\end{gathered}
$$

With each point $(x, y)$ of $E_{4} R_{0}$ is associated a sequence of rectangles satisfying the conditions of the last paragraph; and in particular condition (13). Hence we can apply Vitali's theorem and obtain, by (25) and (16), a finite set of such rectangles $R_{1}, . . R_{n}$, say, not 
A. J. Ward:

touching each other or the sides of $R_{0}$, such that

$$
\begin{gathered}
\sum_{v=1}^{n} \mu\left(R_{v}\right)>(1-\varepsilon) \mu\left(R_{0}\right), \\
\sum_{\nu=1}^{n} F\left(R_{\nu}\right)>(A-1) \eta \sum_{\nu=1}^{n} \mu\left(R_{v}\right)
\end{gathered}
$$

From (13), (15) we see that the principal corners of each $R_{v}$ are points of $E_{8}$. Apply the lemma; we can write

$$
R_{0}=\sum_{\nu=1}^{n} R_{\nu}+\sum_{\nu=1}^{n^{\prime}} R_{\nu}^{\prime}
$$

where each $R_{v}^{\prime}$ has one corner belonging to $E_{3}$. We have therefore, by (12) and (24)

$$
F\left(R_{v}^{\prime}\right) \geqslant-M \mu\left(R_{v}^{\prime}\right)
$$

and so from $(20),(26),(27)$ and $(28)$, since $F(R)$ is additive,

$$
\begin{aligned}
F\left(R_{0}\right) & >(A-1) \eta \sum_{\nu=1}^{n} \mu\left(R_{\nu}\right)-M \sum_{\nu=1}^{n^{\prime}} \mu\left(R_{v}^{\prime}\right) \\
& >(A-1) \eta \mu\left(R_{0}\right)-\left\{M+(A-1) \eta \sum_{\nu=1}^{n^{\prime}} \mu\left(R_{\nu}^{\prime}\right)\right. \\
& >(A-1) \eta \mu\left(R_{0}\right)-2 M \varepsilon \mu\left(R_{0}\right) \\
& >(A-2) \eta \mu\left(R_{0}\right),
\end{aligned}
$$

(since $(A-1) \eta<M)$. This contradicts $(23)$ and so $(7)$ is established.

6. A system of rectangles $R_{n}(x, y)$ associated with a point $(x, y)$ will be said to be completely regular if

(i) Each $R_{n}(x, y)$ contains $(x, y)$;

(ii) $\quad d\left(R_{n}\right) \rightarrow 0$ as $n \rightarrow \infty$;

(iii) There exists $\alpha>0$ such that, for all $n$,

$$
\begin{aligned}
& \mu\left(R_{n}\right)>\alpha\left[d\left(R_{n}\right)\right]^{2}, \\
& d\left(R_{n+1}\right)>\alpha d\left(R_{n}\right) .
\end{aligned}
$$

Theorem 2. If $\underline{D}(x, y)>-\infty$ at each point of a set $E$, then, except at the points of a set $N$ of measure zero [independent of the systems $R_{n}(x, y)$ ], we have at each point of $E$

$$
\varliminf_{n \rightarrow \infty} \frac{F\left[R_{n}(x, y)\right]}{\mu\left[R_{n}(x, y)\right]}=\underline{D}(x, y)
$$

for every completely regular system $R_{n}(x, y)$.
Suppose the theorem false; then there exists a subset $E_{1}$ of $E$, of positive outer measure, with each point of which is associated a completely regular sequence of rectangles $R_{n}(x, y)$ such that

$$
\lim _{n \rightarrow \infty} \frac{F\left[R_{n}(x, y)\right]}{\mu\left[R_{n}(x, y)\right]}>\underline{D}(x, y) \text {. }
$$

The number $\alpha$ occurring in (30) and (31) will depend on $(x, y)$, but we can find a fixed $\alpha$ so small that (30) and (31) are satisfied, for this value of $\alpha$, at all points of a subset $E_{2}$ of $E_{1}$ with positive outer measure. Let $B$ be an integer such that

$$
B a^{3}>576 \text {. }
$$

Arguing as in $\S 3$ we show that there exist $\eta>0, \delta>0$, an integer $A$, and a set $E_{3}$, contained in $E_{2}$, of positive outer measure such that, for $(x, y)$ in $E_{3}, 0<|h|<\delta, 0<|k|<\delta$, we have

and also

$$
\begin{gathered}
A \eta<\underline{D}(x, y) \leqslant(A+1) \eta ; \\
\varliminf_{n \rightarrow \infty} \frac{F\left[R_{n}(x, y)\right]}{\mu\left[R_{n}(x, y)\right]}>(A+B) \eta ; \\
F[R(x, y ; h, k)]>A \eta|h k| ;
\end{gathered}
$$

whenever $d\left(R_{\mu}\right)<\delta$.

Consider a point $\left(x_{0}, y_{0}\right)$ which belongs to $E_{3}$ and is a point of outer density of $E_{3}$ in the strong sense. Then from (33) it follows that we can find $h, k$ such that the rectangle $R_{0}=R\left(x_{0}, y_{0} ; h, k\right)$ satisfies

$$
\begin{gathered}
0<|h|<1, \quad 0<|k|<1 \delta \\
F\left(R_{0}\right)<(A+2) \eta \mu\left(R_{0}\right), \\
\mu_{\prime^{\prime}}\left(E_{3} R_{0}\right)>\mu\left(R_{0}\right)\left(1-\frac{\alpha^{3}}{1000}\right) .
\end{gathered}
$$

It is convenient to suppose $0<h \leqslant k$ (it will readily appear how to apply the argument in the other possible cases); making this supposition we define the integer $m$ such that

$$
m \geqslant 2, \quad m h \leqslant 2 k<(m+1) h .
$$


We consider the $2 m$ squares $S_{p q}$ defined by

$$
\begin{array}{lr}
x_{0}+(p-1) h / 2 \leqslant x \leqslant x_{0}+p h / 2, & (p=1,2) \\
y_{0}+(q-1) h / 2 \leqslant y \leqslant y_{0}+q h / 2, & (q=1,2 \ldots m)
\end{array}
$$

and the two rectangles $R_{p}$,

$$
\begin{gathered}
x_{0}+(p-1) h / 2 \leqslant x \leqslant x_{0}+p h / 2, \quad(p=1,2) \\
y_{0}+m h / 2 \leqslant y \leqslant y_{0}+k,
\end{gathered}
$$

except that if $2 k=m h$ exactly, these rectangles are naturally omitted. A square $S_{p q}$ will be said to be of class $A$ if

$$
\mu_{e}\left(E_{3} S_{p q}\right)>\mu\left(S_{p q}\right)-\alpha^{3} h^{2} / 144 ;
$$

otherwise it will be said to be of class $B$. Let $m^{\prime}$ be the number of squares of class $B$; then clearly we have

$$
\mu_{e}\left(E_{3} R_{0}\right) \leqslant \mu\left(R_{0}\right)-m^{\prime} \alpha^{3} h^{2} / 144
$$

and so from (40) and (41) we obtain

and so

$$
m^{\prime} \alpha^{3} h^{2} / 144<\alpha^{3} h k / 1000<\alpha^{3} h^{2}(m+1) / 2000
$$

$$
\begin{aligned}
m^{\prime} & <144(m+1) / 2000 \\
& <(m+1) / 12 \\
& <m / 6 .
\end{aligned}
$$

From this it follows that each of the two columns of $m$ squares given by $p=1$ and $p=2$ contains some square of class $A$. Then the squares of each column, together with the corresponding rectangle $R_{p}$ (if it exists), can be grouped together into rectangles, each of which contains exactly one square of class $A$. We suppose this process carried out for each column. Of the resulting rectangles, some will consist each of a single square of class $A$; these we enumerate as $S_{1}, S_{2}, \ldots S_{M}$. The remaining rectangles, each of which contains one or more squares of class $B$ or possibly a rectangle $R_{1}$ or $R_{2}$, we enumerate as $R_{1}^{\prime}, R_{2}^{\prime}, \ldots R_{M^{\prime}}^{\prime}$. Clearly $M^{\prime} \leqslant m^{\prime}+2$, and so, since by the construction $M=\left(2 m-m^{\prime}\right)-M^{\prime}$, we have from (41) and (47)

$$
\begin{aligned}
M & >2 m-m / 3-2 \\
& >m / 2 .
\end{aligned}
$$

Now by the additive property of $F(R)$

$$
F\left(R_{0}\right)=\sum_{\nu=1}^{M} F\left(S_{\nu}\right)+\sum_{\nu=1}^{M{ }^{\prime}} F\left(R_{\nu}^{\prime}\right)
$$

We shall obtain a lower bound for each of these terms.

7. Consider a square $S_{\nu}$ (one of the squares $S_{p q}$ ); let $\left(x_{\nu}, y_{\nu}\right)$ be its middle point, so that its principal corners are $\left(x_{\nu}-h / 4, y_{\nu}-h / 4\right)$ and $\left(x_{\nu}+h / 4, y_{\nu}+h / 4\right) . S_{\nu}$ is of class $A$; hence by (46) there is certainly $\left.{ }^{7}\right)$ a point $\left(x_{v}^{\prime}, y_{v}^{\prime}\right)$ of $E_{3}$ such that

$$
\begin{aligned}
& x_{\nu}-h / 12<x_{\nu}^{\prime}<x_{\nu}+h / 12, \\
& y_{\nu}-h / 12<y_{\nu}^{\prime}<y_{\nu}+h / 12 .
\end{aligned}
$$

Let $R_{N}\left(x_{\nu}^{\prime}, y_{v}^{\prime}\right)$ be the first of the sequence of rectangles $R_{n}\left(x_{\nu}^{\prime}, y_{v}^{\prime}\right)$ which satisfies

$$
d\left[R_{n}\left(x_{\nu}^{\prime}, y_{v}^{\prime}\right)\right]<h / 12 ;
$$

then by (31), (36) and (38) we have

$$
\alpha h / 12<d\left[R_{N}\left(x_{\nu}^{\prime}, y_{v}^{\prime}\right)\right]<h / 12,
$$

and so by $(30)$

$$
\mu\left[R_{N}\left(x_{v}^{\prime}, y_{v}^{\prime}\right)\right]>\alpha^{3} h^{2} / 144 .
$$

Let $\left(\xi_{1 v}, \eta_{1}\right)$ and $\left(\xi_{2 n}, \eta_{2 v}\right)$ be the principal corners of $R_{N}\left(x_{v}^{\prime}, y_{v}^{\prime}\right)$, so that, by (50), (51) and (52) we have

$$
\begin{aligned}
& x_{\nu}-h / 6<\xi_{1 \nu}<\xi_{2 \nu}<x_{\nu}+h / 6 ; \\
& y_{\nu}-h / 6<\eta_{1 \nu}<\eta_{2_{\nu}}<y_{\nu}+h / 6 ;
\end{aligned}
$$

also from (52) and (53), since $\mu\left(R_{N}\right)<d\left(R_{N}\right)\left(\xi_{2 v}-\xi_{1 v}\right)$ we have

$$
\xi_{2,}-\xi_{1 v}>\alpha^{3} h / 12 \text {. }
$$

Consider now the four rectangles

$\begin{array}{lll}(57) & x_{\nu}-h / 4<x<\xi_{1 \nu,} & y_{\nu}-h / 4<y<y_{\nu}+h / 4 ; \\ (58) & \xi_{2 v}<x<x_{\nu}+h / 4, & y_{\nu}-h / 4<y<y_{\nu}+h / 4 ; \\ (59) & \xi_{1 \nu}<x<\xi_{2 v}, & y_{\nu}-h / 4<y<\eta_{1 \nu} ; \\ (60) & \xi_{1 \nu}<x<\xi_{2 v}, & \eta_{2 v}<y<y_{\nu}+h / 4 ;\end{array}$

which together with $R_{N}\left(x_{\nu}^{\prime}, y_{v}^{\prime}\right)$ make up the square $S_{v}$. By (54), 
(55) and (56) we see that each of these has area at least $\left(\alpha^{3} h / 12\right)$ $(h / 12)=a^{3} h^{2} / 144 ;$ and so by (46) each rectangle contains a point of $E_{3}$; say $P_{1}, P_{2}, P_{3}, P_{4}$ respectively. It follows that the square $S_{v}$ can be divided into the rectangle $R_{N}\left(x_{\nu}^{\prime}, y_{\nu}^{\prime}\right)$ and sixteen other rectangles, say $R_{v 1}, R_{v 2}, \ldots R_{p, 16}$, each of which has at one corner a point

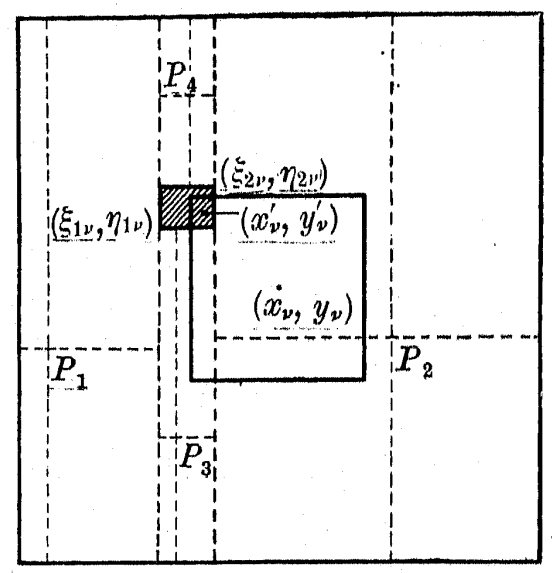

Fig. 1.

of $E_{3}{ }^{8}$ ). The diameters of all these rectangles are less than $h$ and so, by (38), less than $\delta$; hence by (32), (35), (37) and (53) we have

$$
\begin{aligned}
F\left(S_{v}\right) & =F\left[R_{N}\left(x_{v}^{\prime}, y_{v}^{\prime}\right)\right]+\sum_{\lambda=1}^{114} F\left(R_{\nu \lambda}\right) \\
& >(A+B) \eta \mu\left[R_{N}\left(x_{\nu}^{\prime}, y_{\nu}^{\prime}\right)\right]+A \eta \sum_{\lambda=1}^{11} \mu\left(R_{\nu \lambda}\right) \\
& >A \eta \mu\left(S_{\nu}\right)+B \eta \mu\left[R_{N}\left(x_{\nu}^{\prime}, y_{\nu}^{\prime}\right)\right] \\
& >A \eta \mu\left(S_{\nu}\right)+B \eta \alpha^{3} h^{2} / 144 \\
& >A \eta \mu\left(S_{\nu}\right)+4 \eta h^{2} .
\end{aligned}
$$

Consider finally the rectangles $R_{\nu}^{\prime}$; every such rectangle contains: a square of class $A$ and so certainly some point of $E_{3}$. Hence it can be divided into at most four rectangles, each of which has one corner at such a point of $E_{3}$. Their diameters are less than $h+k$ and so less than $\delta$. From (35) we obtain by addition over these sub-rectangles.

$$
F\left(R_{v}^{\prime}\right)>A \eta \mu\left(R_{v}^{\prime}\right)
$$

8) Each of the four rectangles $(57)-(80)$ is divided into four by lines through the points $P_{1}, P_{2}, P_{2}, P_{4}$ respectively.
From (49), (61) and (62) we obtain

$$
F\left(R_{0}\right)>A \eta\left[\sum_{\nu=1}^{M} \mu\left(S_{\nu}\right)+\sum_{\nu=1}^{M^{\prime}} \mu\left(R_{\nu}^{\prime}\right)\right]+4 M \eta h^{2}
$$

and therefore by (39) and (48)

and so

$$
\begin{gathered}
(A+2) \eta \mu\left(R_{0}\right)>F\left(R_{0}\right)>A \eta \mu\left(R_{0}\right)+2 m \eta h^{2} \\
2 \eta h k>2 m \eta h^{2} \geqslant(m+1) \eta h^{2}
\end{gathered}
$$

which contradicts (41). We obtain a similar contradiction if $R_{0}$ has any other possible shape, instead of that given by $0<h \leqslant k$. Hence the theorem is proved.

Corollary 1. If at each point of a set $E$ we have $D(x, y)>-\infty$; then, almost everywhere in $E, \underline{D}(x, y)=\underline{D}_{m}(x, y)$.

It is only necessary to consider the completely regular system $R_{n}(x, y)=R\left(x, y ; 2^{-n}, 2^{-n}\right)$.

Corollary 2. If the function $F(R)$ is of bounded variation, in parti :ular if $F(R)=\iint_{R} f(x, y) d x d y$; then almost everywhere either

or

$$
\underline{D}(x, y)=-\infty
$$

$$
\underline{D}(x, y)=D_{m}(x, y) \text {. }
$$

For in this case it is known that $D_{m}(x, y)$ exists almost everywhere $\left.{ }^{\circ}\right)$.

8. It will be observed that measurability has played no part in the preceding arguments. It is easy to show, however, that $\bar{D}(x, y)$ and $\bar{F}(x, y)$ are measurable functions $\left.{ }^{10}\right)$. This will enable us to prove another theorem similar to Theorem 1 .

Let $\{R\}$ be any collection of closed rectangles, contained in some square; the cardinal number of the collection is quite arbitrary. Then we say that the set $E=\sum R$ is measurable. For with each point of $E$ can be associated a sequence of squares, each containing the point and with diameters tending to zero, which lie in one of the rectangles $R$. By Vitali's theorem, for any positive $\varepsilon$ there is a

9) S. Saks, Theorie de l'Intégrale, p. 49.

10) $\bar{F}(x, y)$ is defined as $\lim _{d(S) \rightarrow 0} \frac{F(S)}{\bar{l}(S)}$ for all squares $S$ containing $(x, y)$; we have olearly $\bar{D}_{m}(x, y) \leqslant \bar{F}(x, y) \leqslant \bar{D}(x, y)$ : s aks, loc. cit. p. 46 . 
finite non-overlapping set of such squares, say $s_{1}, \ldots S_{n}$, such that

$$
\mu\left(\sum_{\nu=1}^{n} S_{n^{\prime}}\right)=\sum_{\nu=1}^{n} \mu\left(S_{n}\right)>\mu_{e}(E)-\varepsilon
$$

But each square $S_{v}$ is included in $E$; and so

$$
\mu_{i}(E) \geqslant \mu\left(\sum_{i=1}^{n} S_{i}\right)>\mu_{\theta}(E)-\varepsilon .
$$

Since $\varepsilon$ is arbitrary, this shows that $E$ is measurable. Let $E(K \delta)$ be the sum of all rectangles $R$ such that $F(R)>K \mu(R)$ and $\delta(R)<\delta$. Then by the above remark $E(K, \delta)$ is measurable. The set of points at which $\bar{D}(x, y) \geqslant K$ is identical with the set $\prod_{n=1}^{\infty} E(K-1 / n, 1 / n)$, and so $\bar{D}(x, y)$ is measurable $\left.{ }^{1 x}\right)$. Again, the set of points $(x, y)$ such that $F(R) \leqslant K_{\mu}(R)$ for any rectangle, containing $(x, y)$, of diameter less than $\delta$, is the complement of $E(K, \delta)$, and therefore is measurable.

9. Theorem 3. ${ }^{12}$ ). If at each point of a set $E$ we have $-\infty<\underline{D}(x, y) \leqslant \bar{F}(x, y)<\infty$; then almost everywhere in $E, \underline{D}(x, y)=$ $=\underline{D}_{m}(x, y)=\bar{D}_{m}(x, y)$.

Suppose the theorem false; then arguing as in the proof of Theorem 1 we find $M>0, \eta>0$, an integer $A, \delta>0$, and a set $E_{3}$ of positive outer measure such that, for $(x, y)$ in $E_{3}$,

(63) $\quad-M<\underline{D}(x, y)<(A-2) \eta<A \eta<\bar{D}_{m}(x, y) \leqslant \bar{F}(x, y)<M$;

$$
-M \mu(R) \leqslant F(R)
$$

whenever $d(R)<\delta$ and $R$ contains $(x, y)$; and finally

$$
F(S) \leqslant M \mu(S)
$$

whenever $d(S)<\delta$ and the square $S$ contains $(x, y)$. Let $H$ be the set of points which satisfy the last two conditions, (64) and (65), but not necessarily (63); then by the preceding arguments $H^{\prime}$ is measurable. It follows that almost all points $\left(x_{0}, y_{0}\right)$ of $H$, and $a$ fortiori almost all points of $E_{3}$, are such that the sub-set of $F$ lying, on the line $y-y_{0}=x-x_{0}$ is linearly measurable and has linear density 1

11) Substituting 'squares' for 'rectangles' we see that $\bar{F}(x, y)$ is measurable. In this case the proof is virtually identical with that given by $\mathrm{S}$ a ks; 1 . cit. p. 47 . covitch. at the point $\left.\left(x_{0}, y_{0}\right){ }^{13}\right)$. Let $E_{4}$ be the set of those points of $E_{3}$ which have this property; then $\mu_{e} E_{4}=\mu_{e} E_{3}>0$. We shall : show that with each point of $E_{4}$ can be associated a sequence of squares $S_{n}(x, y)=$ $=R\left(x, y ; h_{n}, h_{n}\right)$ such that

$$
\begin{gathered}
h_{n} \rightarrow 0 \text { as } n \rightarrow \infty \\
\left(x+h_{n}, y+h_{n}\right) \text { belongs to } H \\
F\left[S_{n}(x, y)\right]>(A-1) \eta h_{n}^{2} .
\end{gathered}
$$

Thereafter the proof proceeds exactly as in $\S 5$, by considering a fixed point $\left(x_{0}, y_{0}\right)$ of $E_{4}$ which is also a point of outer density of $E_{4}$ in the strong sense. ( $H$ takes the place of $E_{3}$ ). It remains only to show how to form the sequences of squares.

10. Let $\left(x_{1}, y_{1}\right)$ be a point of $E_{4}$. From (63) and the fact that $\left(x_{1}, y_{1}\right)$ is a point of linear density of $H$, we can find a sequence of squares $S_{n}^{\prime}=R\left(x_{1}, y_{1} ; l_{n}, l_{n}\right)$ such that

$$
\begin{gathered}
l_{n}<4 \delta \text { and } l_{n \rightarrow 0} \text { as } n \rightarrow \infty \\
F\left(S_{n}^{\prime}\right)>A \eta \eta l_{n}^{2} ; \\
m\left[H ; 0 \leqslant x-x_{1}=y-y_{1} \leqslant 2 l_{n}\right]>(2-\varepsilon) \sqrt{2} l_{n i}
\end{gathered}
$$

where $m$ denotes linear Lebesgue measure ${ }^{14}$ ) and

$$
\varepsilon \leqslant \eta / 8 M<1 \text {. }
$$

Consider one such square $S_{n}^{\prime}$ and suppose for example that $l_{n}>0$. By (71) there exists $h_{n}$ such that

$$
\begin{gathered}
l_{n} \leqslant h_{n}<(1+\varepsilon) l_{n} \text {; } \\
\left(x_{1}+h_{n}, y_{1}+h_{n}\right) \quad \text { belongs to } H .
\end{gathered}
$$

Then $S_{n}\left(x_{1}, y_{1}\right)$ is the square $R\left(x_{1}, y_{1} ; h_{n}, h_{n}\right)$. (If $l_{n}<0$ we proceed similarly; the inequalities in (73) are reversed). To show that this sequence of squares satisfies the required conditions it remains only to prove (68).

Let $R_{n 1}, R_{n 2}$ be the rectangles, respectively.

$$
x_{1}+l_{n} \leqslant x \leqslant x_{1}+h_{n} ; \quad y_{1} \leqslant y \leqslant y_{1}+h_{n}
$$

13) Saka, loc. cit. p. 226 .

14) It is clear that if $l_{n}$ is negative we must read $m\left[H ; 0 \geqslant x-x_{1}=y-y_{1} \geqslant 2 l_{n}\right]$ in (71). 


$$
x_{1} \leqslant x \leqslant x_{1}+h_{n, \quad} \quad y_{1}+l_{n} \leqslant y \leqslant y_{1}+h_{n}
$$

and let $S_{n}^{\prime \prime}$ be the square

$$
x_{1}+l_{n} \leqslant x \leqslant x_{1}+h_{n}, \quad y_{1}+l_{n} \leqslant y \leqslant y_{1}+h_{n}
$$

Since $F(R)$ is additive we have

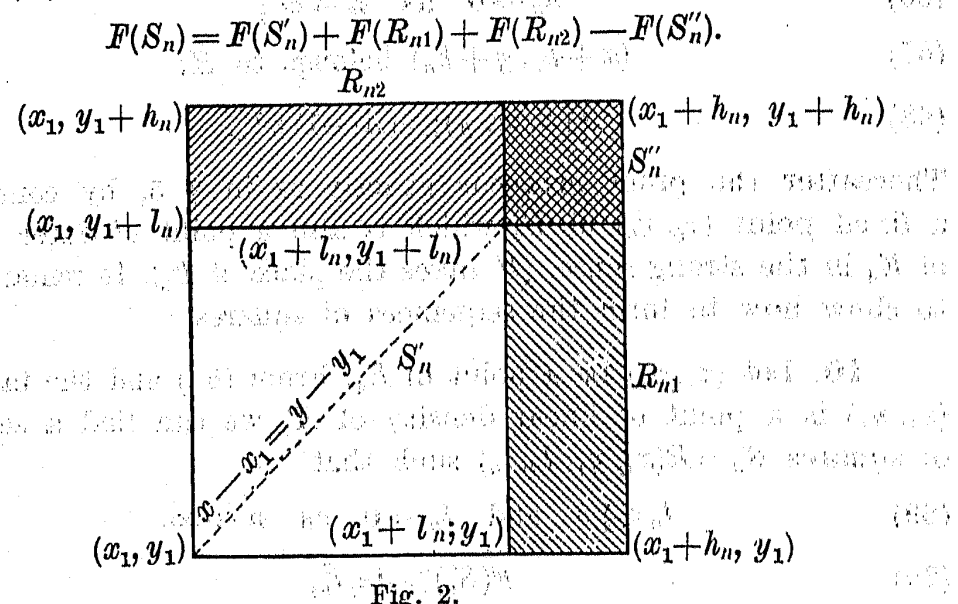

Since the point $\left(x_{1}+h_{n}, y_{1}+h_{n}\right)$ belongs to $H$ and is a corner of edch of the rectangles $R_{m 1}, R_{m i 2}, S_{\mu}^{\prime \prime}$ we can apply $(64)$ and $(65)$; from (73) and (78) we derive thus

$$
\begin{aligned}
& \left.F\left(S_{n}\right) \geqslant F\left(S_{n}^{\prime}\right)-M\left(\mu R_{n 1}\right)+\mu\left(R_{n 2}\right)+\mu\left(S_{n}^{\prime \prime}\right)\right\} \\
& \geqslant F\left(S_{n}^{\prime}\right)-M l_{n}^{\prime} \varepsilon(2+3 \varepsilon) \\
& (70),(72),(73) \text { and }(79) \text { we obtain finally }
\end{aligned}
$$

From (63), (70), (72), (73) and (79) we obtain finally

$$
\begin{aligned}
F\left(S_{n}\right) & >A \eta l_{n}^{2}-5 M l_{n}^{2} \varepsilon \\
& >A \eta h_{n}^{2}-5 M l_{n}^{2} \varepsilon-M\left(h_{n}^{2}-l_{n}^{2}\right) \\
& \left.>A \eta h_{n}^{2}-5 M l_{n}^{2} \varepsilon-M l_{n}^{2} \varepsilon^{2}+2 \varepsilon\right) \\
& >A \eta h_{n}^{2}-8 M l_{n}^{2} \varepsilon \\
& >A \eta h_{n}^{2}-8 M h_{n}^{2} \varepsilon \\
& >(A-1) \eta h_{n}^{2}
\end{aligned}
$$

which is the result (68) required. The argument is practically the same in the case when $l_{n}$ and $h_{n}$ are negative. ${ }^{15}$ ).

15) If $F(x, y)$ is continuous we can replace the condition $\bar{T}(x, y)<\infty$, in Theorem 3 , by the weaker condition $\bar{D}_{m}(x, y) \cdots \infty$. The argument is unchanged, except that $(65)$ is replaced by the condition (65 A): $F[R(x, y ; h, h)] ; M h^{2}$ whenever $|h| \delta$ and $(x, y)$ is in $E_{3}$ : The set of points satisfying $(65 \mathrm{~A})$ is closed, and so $H$ is measurahle.
11. For measurable functions of one variable, there is a theorem which states that if any of the four derivates is finite in a set $E$, then it is the approximate derivative almost everywhere in $E$. An analogous theorem, in the weak sense, can be proved for functions of rectangles, but the corresponding theorem in the strong sense is false. Let $\left(x_{1}, y_{1}\right)$ be a point such that $D\left(x_{1}, y_{1}\right)>-\infty$. Given $\eta>0$, we write $G\left(x_{1}, y_{1}, \eta\right)$ for the set of points $(x, y)$ such that

(80) $\left|\Delta F\left(x_{1}, y_{1} ; x-x_{1}, y-y_{1}\right)-\left(x-x_{1}\right)\left(y-y_{1}\right) D\left(x_{1}, y_{1}\right)\right|>$

$$
\left.>\eta \mid\left(x-x_{1}\right)\left(y-y_{1}\right)\right) \text {. }
$$

Theorem 4. If at each point of a set $E \underline{D}(x, y)>-\infty$, then almost everywhere in $E$, for all $\eta>0$,

$$
\lim _{d(S) \rightarrow 0} \frac{\mu_{e}[S E G(x, y, \eta)]}{\mu S}=0,
$$

where $\mathbb{S}$ is any square containing $(x, y)$.

Suppose the theorem untrue; then by the usual arguments we can find fixed $\varepsilon>0, \eta>0$, an integer $A, \delta>0$, such that, in a subset $E_{1}$ of $E$ with positive outer measure we have

$$
\begin{gathered}
\varlimsup_{d(S) \rightarrow 0} \frac{\mu_{e}[S E G(x, y, 3 \eta)]}{\mu S}>8 \varepsilon \\
A \eta<\underline{D}(x, y) \leqslant(A+1) \eta
\end{gathered}
$$

if $d(R)<\delta$ and $R$ contains $(x, y)$.

Let $H$ be the set of points $(x, y)$ satisfying the last condition, (83); then $H$ is measurable and contains $E_{1}$. Hence $(E-H)$ has zero density at almost all points of $E_{1}$, say at the points of a set $E_{2}$. Thus we have, if $(x, y)$ is in $E_{2}$,

$$
\varlimsup_{\alpha(S) \rightarrow 0} \frac{\mu_{e}[S H G(x, y, 3 \eta)]}{\mu \mathcal{S}}>8 \varepsilon
$$

Now consider any square $S$ containing $(x, y)$, in which the average outer density of the set $H G(x, y, 3 \eta)$, (that is, the quotient occurring in (84)), is greater than $8 \varepsilon$. Let $S_{0}$ be the smallest square with centre $(x, y)$ and containing $s$; then $\mu S_{0} \leqslant 4 \mu S$ and so the average density of the set in $S_{0}$.is greater than $2 \varepsilon_{\text {. Divide }} S_{0}$ by lines 
through $(x, y)$ into four equal squares; in one of these at least the average density is greater than $2 \varepsilon$. It follows that at each point of $E_{2}$, either:

$$
\begin{aligned}
& \text { either } \\
& \text { (85) } \lim _{h \rightarrow 0} \frac{\mu_{c}[H G(x, y, 3 \eta) R(x, y ; h, h)]}{h^{2}}>2 \varepsilon \\
& \text { or } \quad \lim _{h \rightarrow 0} \frac{\mu_{e}[H G(x, y, 3 \eta) R(x, y ; h,-h)]}{h^{2}}>2 \varepsilon
\end{aligned}
$$

Hence one at least of $(85)$ and (86) must be true in a set $E_{3}$ of positive outer measure; and we may suppose it is (85), for in the other case we could consider $\Phi(x, y)=-F(x,-y)$. . . .

Let $\left(x_{1}, y_{1}\right)$ be a point of $\boldsymbol{E}_{3}$. There is a sequerice $\eta_{m},\left|l_{n}\right|<1, \delta$, $l_{n} \rightarrow 0$, such that

$$
\frac{\mu_{e}\left[H G\left(x_{1}, y_{1}, 3 \eta\right) R\left(x_{1}, y_{1} ; l_{n}, l_{n}\right)\right]}{l_{n}^{2}}>2 \varepsilon .
$$

Hence for each $n$ there is a point $\left(x_{1}+h_{n}, y_{1}+k_{n}\right)$, say, lying in $H G\left(x_{1}, y_{1}, 3 \eta\right) R\left(x_{1}, y_{1} ; l_{n}, l_{n}\right)$ and such that

$$
h_{n}|>\varepsilon| l_{n}|, \quad| k_{n}|>\varepsilon| l_{n} \mid
$$

It follows that

$$
h_{n} k_{n}>0, \quad \varepsilon\left|h_{n}\right|<\left|k_{n}\right|<\varepsilon^{\prime}\left|h_{n}\right|
$$

and from $(80)$

$$
F\left[R\left(x_{1}, y_{1} ; h_{n}, k_{n}\right)\right]-\underline{D}\left(x_{1}, y_{1}\right) h_{n} k_{n} \mid>3 \eta h_{n} k_{n}
$$

Comparing this with (82) and (83) we have

$$
F\left[R\left(x_{1}, y_{1} ; h_{n}, k_{n}\right)\right]>(A+3) \eta h_{n} k_{n}
$$

We note finally that $\left(x_{1}+h_{n}, y_{1}+k_{n}\right)$ belongs to $H$. From this point the argument is exactly parallel to those used in proving Theorems 1 and 3 ; the detail is left to the reader.

Note. The theorem obtained by replacing ,any square" by ,any" rectangle" in the enunciation of Theorem 4 is certainly false; this may be seen by considering the case of appositive function of rectangles (for example $f f(x ; y) d x d y$ ) which has at a set of positive mear.: sure $\vec{D}(x, y)=+\infty, D(x, y)$ fnite.

\section{Sur les théorèmes de séparation dans la Théorie des ensembles.}

Par

Casimir Ku ratowski (Warszawa).

Soit $\boldsymbol{A}$ une famille' de sous-ensembles d'un espace composé d'éléments arbitraires. On dit que cette famille satisfait au premier thérème de séparation, lorsqu'à chaque couple d'ensembles disjoints $A_{1}$ et $A_{2}$ appartenant à $A$ correspond un ensemble $B$ qui, ainsi que son complémentaire, appartient à $\boldsymbol{A}$ et qui satisfait aux formules $A_{1} \subset B$ et $B A_{2}=0$. Le deuxième théorème de séparation est satisfait, lorsqu'à chaque couple d'ensembles $A_{1}$ et $A_{2}$ appartenant à $\boldsymbol{A}$ correspond un couple d'ensembles disjoints $C_{1}$ et $C_{2}$ dont les complémentaires appartiennent à $A$ et qui remplissent les formules $A_{1}-A_{2} \subset C_{1}$ et $A_{2}-A_{1} \subset C_{2}$.

Des exemples surtout importants de familles satisfaisant aux deux théorèmes de séparation présentent: la famille des ensembles boreliens de classe multiplicative $\beta>0{ }^{1}$ ), celle des ensembles analytiques ${ }^{2}$ ), celle des ensembles projectifs de classe $C P\left(A^{3}\right.$ ).

1) c. à d. $G_{\delta}, F_{\sigma \delta}, G_{\delta \sigma \delta}$ etc. Les théorèmes de séparation pour ces familles d'ensembles ont été démontrés par MM. Lusin et Sierpiński. Voir W. Sierpiński, Fund. Math. 6 (1924), p. 2 et Bulet. Soc. St. de Cluj 6 (1932), p. 461, et N. Lusin, Fund. Math. 16 (1930), pp. 57 et 60.

Il importe de remarquer que la famille des ensembles fermés satisfait au deuxième théorème de séparation et cependant - si l'espace est connexe - elle ne satisfait pas au premier: à chaque couple $E_{1}, E_{2}$ d'ensembles fermés correspond un couple $H_{1}, H_{2}$ d'ensembles ouverts disjoints tel que $E_{1}-E_{2} \subset H_{1}$ et $E_{2}-E_{1} \subset H_{2}$ (cf. par exemple ma Topologie I. p. 99, 2 et 6), tandis que l'espace ne contient aucun vrai gous-ensemble non vide qui soit simultanément fermé et ouvert.

2) Les théorèmes de séparation pour les ensembles analytiques ont été démontrés par M. Lusin sous le nom du "premier et deuxième principes". Ils jouent un role fondamental dans la théorie de ces ensembles.

3) Les théorèmes de séparation pour les ensembles $C P C A$ ont été démontrés par M. N ovik off, Fund. Math. 25 (1935), p. 459. 